\title{
Priming semántico directo y mediado orto- fonológicamente en pacientes con enfermedad de Parkinson: un estudio de tiempos de reacción
}

\section{Direct and ortho-phonologically mediated semantic priming in patients with Parkinson's disease: Evidence from reaction times}

\author{
Abraham Novoa \\ Departamento de Español, \\ Universidad de Concepción \\ Mónica Veliz \\ Departamento de Español, \\ Universidad de Concepción \\ Ernesto Guerra \\ Instituto de Educación, \\ Centro de Investigación Avanzada \\ (CIAE), \\ Universidad de Chile
}

Contacto con el autor: Abraham Novoa

Concepción - Chile Correo-e: abrahamnovoa@udec.cl

Recibido: $23 / 09 / 2019$ Aceptado: $18 / 11 / 2019$
RESUMEN

El presente estudio aborda el impacto de la enfermedad de Parkinson en el procesamiento de información semántica durante una tarea de reconocimiento visual de palabras. Para ello, se comparó el rendimiento de un grupo de pacientes con Parkinson con el rendimiento de sujetos sin daño neurológico en dos experimentos. Se evaluaron los efectos de tiempo de presentación de un prime semántico directo (Experimento 1) y un prime semántico mediado orto-fonológicamente (Experimento 2). Además, se evaluó la influencia de la frecuencia léxica tanto del prime como la palabra target, sobre el acceso léxico. Los participantes estaban divididos en tres grupos (grupo clínico, grupo de control etario, y grupo de adultos jóvenes). Los tres grupos completaron una tarea de decisión léxica de tiempo de reacción en la modalidad go-no-go. Los participantes debían responder presionando una tecla del computador si el estímulo presentado en la pantalla era una palabra. En el caso contrario cuando era una pseudo palabra, los participantes no debían presionar ninguna tecla. Los resultados en base al número de respuestas correctas y los tiempos de reacción muestran dificultades de procesamiento semántico en el grupo clínico. Sin embargo, este grupo mostró ser sensible a la frecuencia de las palabras y los tiempos de presentación de estas. En su conjunto, los resultados son coherentes con la literatura existente, y contribuyen a comprender de mejor manera el déficit lingüístico presente en la enfermedad de Parkinson.

Palabras clave: enfermedad de parkinson, acceso al léxico, procesamiento semántico, priming léxico, tarea go-no-go.

\section{ABSTRACT}

The present study addresses the incidence of Parkinson's disease in the processing of semantic information during visual word recognition tasks. In order to do so, the performance of a group of patients with Parkinson's was compared with the performance of subjects without neurological impairment in two experiments. We evaluate the effects of presentation time of a semantic prime (Experiment 1 ) and an ortho-phonologically mediated semantic prime (Experiment 2), and the impact of the lexical frequency of both the prime and the target words. Participants divided into three groups (a clinical group, an age control group and a group of young adults), completed a reaction time lexical decision task in the go-no-go modality. They had to respond by pressing a computer key if the stimulus presented on the screen was a word. Otherwise, the participants should not press any key. The results, based on response accuracy and reaction times, show a semantic deficit in the clinical group, while this group was, nevertheless, sensitive to the frequency of the words and their presentation time. As a whole, the results are consistent with the existing literature, and contribute to a better understanding of the linguistic deficit present in Parkinson's disease.

Keywords: parkinson's disease, lexical access, semantic processing, lexical priming, go no go task. 


\section{Introducción}

La enfermedad de Parkinson (EP) es un desorden neurodegenerativo heterogéneo, caracterizado principalmente por síntomas motores y cognitivos (Tirapu, Muñoz y Peregrín, 2002; Tirapu y Muñoz, 2005; Helimch, Hallet, Deushl, Toni y Bloe, 2012; Ting Lu et al., 2016). Diferentes estudios han mostrado que esta enfermedad puede también afectar el procesamiento del lenguaje en distintos niveles (Bastiaanse \& Leenders, 2009; Colman \& Bastiaanse, 2011; Altmann \& Troche, 2011; Illes et al., 1988; Walsh \& Smith, 2012), entre los cuales el acceso léxico se ha descrito como particularmente afectado (Angwin, Chenery, Copland, Murdoch \& Silburn, 2005, 2007). Las alteraciones en el lenguaje podrían estar asociadas, de acuerdo a estudios existentes, al deterioro cognitivo (Grossman, 1999; Angwin et al., 2006; Bastiaanse \& Leenders, 2009; Zanini, Tavano \& Fabbro, 2010). Sin embargo, algunos autores han abogado por un déficit lingüístico independiente, asociado a la denervación de zonas corticales y subcorticales especializadas en el procesamiento del lenguaje (Nadeau, 2008; Colman \& Bastiaanse, 2011). Más allá del debate sobre las bases neurales de este déficit, la evidencia muestra que el acceso léxico se encuentra alterado en la EP y, por lo tanto, entender los mecanismos cognitivos que se encuentran afectados se torna relevante.

Acceder al léxico de entrada, es decir, comprender una palabra, implica comparar un estímulo (visual o auditivo) con sus respectivas representaciones almacenadas en la memoria a largo plazo (Álvarez, De Vega y Carreiras, 1998; Cuetos, González \& De Vega, 2018). Estas representaciones incluyen el almacenamiento del significado, de sus formas fonética-grafémica (Garman, 1995), así como también el acceso a otros significados relacionados. En este sentido, la literatura existente sugiere que los pacientes con EP pueden presentar alteraciones en el acceso al léxico. En particular, en el reconocimiento visual de palabras, y el acceso a su significado, así como en tareas de categorización y jerarquización de conceptos (Portin, Laatu, Revonsuo \& Rinne, 2000; Angwin et al., 2005; Elorriaga et al., 2012; Cardona et al., 2013).

Gran parte de la evidencia empírica en relación con las dificultades de acceso léxico en los pacientes de con EP proviene de estudios de priming (Fischler, 1997). En estas tareas, a los participantes se les presentan pares de palabras en secuencia, que en el caso del priming semántico, están asociadas en su significado (e.g., "cumbre”, "Alpes"). Por lo general, a los participantes se les instruye a tomar una decisión sobre la segunda palabra (e.g., categorización semántica, decisión léxica) presionando un botón. De esta manera, se logra capturar el tiempo de respuesta de la decisión del participante, y por ende el tiempo que le toma acceder al significado de la palabra. Se espera en estos estudios, que la exposición a la primera palabra (o prime), facilite el reconocimiento de la palabra objetivo (o target) cuando estas están asociadas. Finalmente, el tiempo que separa la presentación de estas palabras (o SOA por su nombre en inglés, stimulus onset asynchrony), es manipulado para generar condiciones de priming automático $(\leq$ $250 \mathrm{~ms}$ ) o controlado (> $250 \mathrm{~ms}$ ), dependiendo si el acceso léxico al prime se hace consciente o no.

En esta línea de investigación, el estudio de Angwin, Chenery, Copland, Murdoch y Silburn (2005) 
investigó la integridad de la activación semántica en el reconocimiento visual de palabras en pacientes con $E P$, a través de una tarea de multipriming. Esta tarea conlleva la presentación de dos estímulos prime de manera consecutiva antes de que el participante realizara el juicio de decisión léxica sobre el target (verano-nieve-invierno). Bajo este paradigma se buscó precisar si la activación semántica persistía pese a que uno de los estímulos presentados como prime no estaba asociado semánticamente con el estímulo target. Además, este experimento incluyó distintos intervalos de tiempo (SOAs) entre los estímulos para precisar el tipo de procesamiento asociado a la tarea (automático o controlado). De acuerdo con sus resultados, los pacientes con EP presentaron tiempos de reacción más prolongados en intervalos de $250 \mathrm{~ms}$, cuando la condición de los estímulos prime era relacionado y no relacionado consecutivamente antes del target, lo que indicaría una disrupción de la activación durante el procesamiento automático del significado en esa condición. Sin embargo, cuando el intervalo entre el prime y el target fue de 1200 ms, los efectos de priming desaparecieron. Esto sugiere un deterioro de los procesos de acceso al léxico conscientes.

En una línea similar, otro estudio (Angwin, Copland, Chenery, Murdoch \& Silburn, 2006) examinó el reconocimiento visual de palabras a través del mismo paradigma de multipriming. Al igual que en el estudio de Angwin et al. (2005), se presentaron dos estímulos prime de manera consecutiva antes de realizar el juicio de decisión léxica sobre la palabra target, considerando como variables el tipo de relación semántica entre prime y target (asociado o no asociado) y el tipo de SOA (250 ms y $1200 \mathrm{~ms}$ ). Sin embargo, a diferencia de Angwin et al. (2005), este estudio comparó participantes en estado on (cuando los pacientes están bajo el efecto de Levadopa) y off (cuando los pacientes presentan la sintomatología típica de la enfermedad, sin efecto de Levadopa).

Los resultados del estudio de Angwin et al. (2006) mostraron diferencias solo en el SOA de corta duración (250 ms) al comparar a los pacientes en estado on y off. Los pacientes en estado on presentaron patrones similares de respuesta al grupo control etario en esta condición. No obstante, los pacientes en estado off presentaron latencias de respuestas más prolongadas. Ello indicaría el compromiso de aspectos automáticos, por sobre los conscientes, en el reconocimiento visual de palabras como consecuencia de la modulación dopaminérgica.

En un estudio de seguimiento, Angwin, Chenery, Copland, Murdoch y Silburn (2007) volvieron a evaluar el acceso léxico en pacientes con EP, esta vez con una tarea de estándar de priming (i.e., decisión léxica; pares de palabras). Los participantes debían decidir sobre la palabra target después de la presentación del prime relacionado semánticamente (o no) con un intervalo temporal variable entre el prime y el target (500 ms, 1000 ms, 1500 ms). Bajo estas condiciones de SOA, Angwin et al. (2007) esperaban que los pacientes con EP tuvieran latencias de respuesta más breves en la condición de SOA corto a diferencia de los SOAs de mayor duración en donde se esperaban tiempos más prolongados o una menor facilitación en el juicio de decisión léxica. Los resultados del estudio de Angwin et al. (2007) mostraron que la activación semántica en pacientes con EP decaía en intervalos de larga duración (acceso al léxico consciente), aunque con una magnitud 
variable y podía depender de otros factores como el nivel de comprensión general del lenguaje.

Efectos similares en SOAs de larga duración se han encontrado en otros estudios que han utilizado el paradigma de priming. Por ejemplo, el estudio Castner et al. (2007) utilizó un SOA de corta duración de 200 ms y un SOA de larga duración de 1000 ms en una tarea de decisión léxica. Los resultados de Castner et al. (2007) indican que los pacientes con EP presentaban latencias más prolongadas (en comparación con un grupo control) en la condición de SOA de larga duración, pero no así en la condición de SOA corto. Este resultado, nuevamente revelaría que los pacientes manifiestan mayor dificultad con el acceso léxico cuando el procesamiento semántico es consciente.

Otro estudio con la misma metodología, (Copland, Sefe, Ashley, Hudson \& Chenery, 2009) usó la tarea para analizar la capacidad de los pacientes con EP de inhibir información semántica. Copland et al. (2009) utilizaron el significado de palabras polisémicas para la condición de prime y las clasificaron según la dominancia del significado en relación al target (e.g., banco - río; banco - dinero). Asimismo, los autores manipularon el tipo de SOA de acuerdo con su duración; el SOA de corta duración fue de 200 ms y el SOA de larga duración fue de 1200 ms. Los resultados del estudio mostraron que los pacientes con EP presentan una menor capacidad para suprimir el significado relevante en el SOA de larga duración a diferencia del grupo control etario. Esto ha sido interpretado, una vez más, como una dificultad en la selección del significado cuando el significado del prime es consciente.
Un estudio anterior a Copland et al. (2009), ya había investigado la capacidad de los pacientes de inhibir información irrelevante al momento de realizar el juicio de lexicalidad sobre el estímulo target (Marí-Beffa, Hayes, Machado \& Hindle, 2005). Para ello, se consideró como índice los estímulos usados como distractores (pseudopalabras) y los pares de palabras que no estaban asociadas semánticamente, ya que estas permiten evaluar la capacidad de los participantes de suprimir una respuesta en función del juicio de lexicalidad. Los resultados del estudio de Marí-Beffa et al. (2005) mostraron que los pacientes con EP presentaban una menor capacidad de inhibición de respuestas comparados con un grupo de control etario. Esto generaría un efecto de hiperpriming, favoreciendo los tiempos de reacción en los pares de palabras asociadas semánticamente. Lo anterior se traduce en que los pacientes con EP presentan dificultades para discriminar los pares de palabras que no están relacionadas semánticamente.

En suma, el conjunto de evidencia descrita sugiere que los pacientes con EP presentan un procesamiento de acceso léxico alterado, ya que los efectos de priming semántico difieren de aquellos observados en grupos controles de participantes sanos. En particular, la evidencia sugiere que el acceso a información irrelevante (prime) y la incapacidad de inhibirlo de manera oportuna, genera tiempos de reacción más largos. Principalmente, cuando se utilizan intervalos de larga duración, es decir, en acceso el léxico de tipo consciente.

Sin embargo, poco se sabe del rol de otras variables que pudiesen facilitar o inhibir la activación de información como es el caso de la frecuencia léxica o la mediación orto-fonológica del acceso semántico, 
esta última entendida como la relación entre un vecino orto-fonológico (e.g., lumbre) de otra palabra (e.g., cumbre) asociada en significado a una tercera palabra (e.g., Alpes), y por tanto como mecanismo indirecto de facilitación en el acceso léxico. La frecuencia de las palabras es una variable léxica que refleja la cantidad de veces que aparece una palabra en el lenguaje compartido por una comunidad lingüística. En adultos el efecto de la frecuencia léxica es muy robusto en una variedad de tareas léxicas que involucran palabras escritas, siendo las palabras de alta frecuencia las que presentan una ventaja de procesamiento (Balota \& Chumbley, 1984; Monsell, 1991; Rayner y Duffy, 1986; ver Brysbaert, Mandera \& Keuleers, 2018, para revisión reciente).

Por su parte, la mediación orto-fonológica de los efectos de priming semántico ha demostrado modificar los patrones de acceso léxico en tareas de priming en participantes jóvenes en función del tiempo de presentación (SOA), es decir, si el acceso es consciente o no (Carreiras \& Perea, 2002; Duñabeitia, Carreiras \& Perea, 2008). Dado que los pacientes con EP muestran más dificultad con procesos conscientes (comparados con grupos control), se hace relevante evaluar este nivel de mediación indirecta.

La pregunta central del presente estudio surge de la necesidad de precisar de qué manera la EP incide en el procesamiento léxico mediado directamente (Experimento 1) e indirectamente (Experimento 2) por relaciones semánticas a nivel automático (SOA de corta duración) y consciente (SOA de larga duración), así como los efectos de la variación de frecuencia léxica. Esta pregunta no ha sido previamente investigada en hablantes de castellano, lengua en la cual la incidencia de factores orto-fonológicos es predominante (Duñabeitia, Carreiras \& Perea, 2008).

Para ello, se diseñaron dos experimentos conductuales de tiempos de reacción. La modalidad de la tarea, para ambos experimentos, consiste en una tarea go-no-go. Los participantes debían responder, presionando una tecla, cuando consideraran que el estímulo presentado era una palabra y suprimir su respuesta cuando consideraran que el estímulo no correspondía a una palabra (pseudopalabra). Tanto en el experimento 1 como en el experimento 2, el intervalo temporal, entre el prime y el target, fue el mismo, considerando el tipo de SOA (de corta duración y de larga duración). Ello para analizar el procesamiento automático y controlado en la activación de información semántica durante una tarea de reconocimiento visual de palabras.

En el primer experimento, se utilizaron estímulos relacionados semánticamente de manera directa (e.g., cumbre - ALPES). Se espera que el desempeño sea peor en comparación a los participantes sin EP. Además, se espera que los pacientes tengan un mejor desempeño, tanto en términos de acierto como de rapidez de respuesta, frente a palabras de alta frecuencia (vs. baja frecuencia). En el segundo experimento, los estímulos estaban relacionados por un vecino orto-fonológico del estímulo prime del primer experimento, manteniendo el mismo estímulo target (e.g., lumbre - ALPES). Se espera, en la tarea de priming mediado orto-fonológicamente un menor efecto de facilitación semántica indirecta en los pacientes con EP, en particular en la condición de SOA de corta duración (Perea \& Rosa, 2000; Duñabeitia, Carreiras \& Perea, 2008). 


\section{Metodología}

\section{Participantes}

La muestra estuvo compuesta, para ambos experimentos, de un total de 100 hablantes nativos de castellano chileno, con visión normal o corregida, divididos en tres grupos. Un grupo experimental compuesto por 20 pacientes diagnosticados con EP con una edad promedio $(\mathrm{m})$ de 70.5 y una desviación estándar (de) de 7.3; un grupo control de adultos mayores saludables $(n=20 ; m=70.7 ; d e=7.3)$ y un grupo control de adultos jóvenes ( $n=60 ; m=20.6$; de=2.8). Cada participante fue informado de los objetivos generales del estudio y de la voluntariedad de la participación a través de un consentimiento informado. La investigación fue aprobada por el Comité de Bioética de la Universidad de Concepción. Una descripción completa del perfil cognitivo de los pacientes con EP se entrega en el Apéndice A.

\section{Materiales y diseño}

Los materiales de los dos experimentos estuvieron compuestos por total de 270 palabras de la base de datos B-Pal (Davis \& Perea, 2005), las que se agruparon en 90 pares, manteniéndose constante la palabra target entre los experimentos (ver Tabla B1, Apéndice B). De igual forma, se crearon 180 pseudopalabras necesarias para la tarea de discriminación léxica agrupadas en 90 pares para cada experimento (Tabla B2, Apéndice B). Dichas palabras se elaboraron invirtiendo las letras de las palabras experimentales. Además, se aseguró que no fueran similares en forma a las palabras estimulares, pero que sí tuvieran una estructura silábica acorde al español para garantizar su lectura, lo que ha sido utilizado clásicamente en estudios de decisión léxica (Perea \& Rosa, 2000; Duñabeitia, Carreiras \& Perea, 2008).

En el experimento 1, los estímulos experimentales fueron seleccionados de acuerdo con la relación semántica entre el prime y el target (e.g., planta HIERBA, respectivamente). El grado de asociación entre ellos se precisó a través de un estudio normativo con una escala tipo Likert en un grupo de 62 estudiantes de la Universidad de Concepción. Los estudiantes debían responder en una escala de 1 a 9 (donde 1 = muy en desacuerdo y 9 = muy de acuerdo) si los pares de palabras estaban asociados semánticamente (ver Tabla B1).

En la mitad de los ítems se consideró un prime de alta frecuencia léxica, mientras que en la otra mitad se consideró un prime de baja frecuencia léxica. La frecuencia del prime $(m=43.4 ; d e=21.8)$, sin embargo, fue siempre más alta que la frecuencia del target ( $m=13.04 ; d e=12.1)$, considerando el criterio de estudios previos para asegurar el efecto de priming (Duñabeitia, Carreiras \& Perea, 2008). De manera similar, las palabras target fueron también divididas en palabras de alta y baja frecuencia léxica. El diseño experimental cruzó dos tiempos de presentación del prime o SOAs (i.e., $80 \mathrm{~ms}$ y $360 \mathrm{~ms}$ ) en dos listas experimentales, utilizando un cuadrado latino. De esta manera, cada ítem apareció en una lista con un SOA de 80 y en otra con uno de 360 ms. Todos los participantes vieron todos los ítems experimentales e igual número de repeticiones en cada una de los dos SOAs.

El segundo experimento fue idéntico al experimento 1 en cuanto a participantes y diseño. Sin 
embargo, las palabras que actuaron como prime fueron distintas. Para el experimento 2, se seleccionó un nuevo set de 90 palabras de la base de datos B-Pal (Davis \& Perea, 2005) las cuales fueran vecinas ortográficas de las palabras que actuaron como prime en el experimento 1 (planta - HIERBA en el experimento 1; llanta - HIERBA en el experimento 2). Como en el primer experimento, las palabras prime se dividieron en alta y baja frecuencia léxica y silábica. Las palabras target fueron las mismas, y por tanto, se mantuvieron los distintos niveles de frecuencia léxica y silábica.

\section{Procedimiento}

La aplicación de los experimentos se realizó en momentos diferentes (un mes de diferencia entre la aplicación del experimento 1 y el experimento 2) para evitar efectos de repetición, considerando que los estímulos targets ni las pseudopalabras no variaron entre experimentos. En ambos experimentos, la presentación de los estímulos se realizó en un computador con el programa E-prime (Versión 3.0). Los participantes fueron instruidos para realizar una tarea de reconocimiento visual de palabras y fueron asignados a una de las dos listas experimentales las que cruzaron los SOA a nivel de ítem. En cada trial experimental, los participantes vieron dos palabras de manera secuencial, siempre la palabra prime precediendo a la palabra target. El tiempo entre ambas palabras estuvo determinado el SOA (i.e., 80 ms y $360 \mathrm{~ms})$, mientras que la duración de presentación de la palabra target estuvo dada por el tiempo de respuesta de los participantes. Ellos debían presionar una tecla (barra espaciadora) cuando la segunda palabra presentada fuese una palabra real en castellano o, en caso contrario (presentación de una pseudopalabra), los participantes debían evitar presionar la barra espaciadora. Se registraron los tiempos de reacción y los errores de cada uno de los participantes. La Figura 1 presenta una descripción gráfica de la tarea go-no-go.

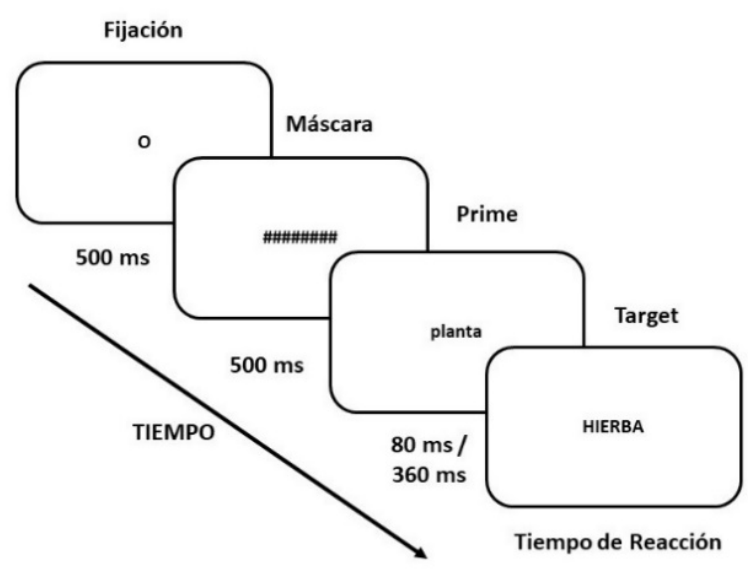

Figura 1. Secuencia de presentación de estímulos.

\section{Análisis de datos}

En ambos experimentos se calculó, para cada participante, el grado de acierto en la tarea, previo al análisis estadístico. De esta manera se verificó que los participantes tuviesen un porcentaje mayor a $65 \%$ de acierto, lo cual garantizaría un mínimo de atención a la tarea. Posteriormente, se eliminaron los datos con respuestas menores de 100 ms o mayores de 1500 ms. Este criterio es utilizado clásicamente por estudios en decisión léxica para excluir respuestas que implican, por un lado, la adivinación de las respuestas cuando los tiempos de reacción son inferiores a $100 \mathrm{~ms}$. Por otro lado, cuando se pierde la atención a la tarea en intervalos superiores a 1500 ms (Perea \& Rosa, 2000; Duñabeitia, Carreiras \& Perea, 
2008). Finalmente, los tiempos de reacción fueron transformados logarítmicamente para ajustarlos más fielmente a una distribución normal. El análisis estadístico se realizó mediante el uso de modelos de regresión con efectos mixtos cruzados, con modelo lineal para los tiempos de reacción y modelo lineal generalizado para los datos de porcentajes de acierto. Para esto se utilizaron los paquetes Ime4 (Bates, Maechler, Bolker, \& Walker, 2015) y ImerTest (Kuznetsova, Brockhoff, \& Christensen, 2017) del software estadístico R (R Core Team, 2017). Dichos modelos permiten acomodar la variabilidad intrínseca a nivel de participante e ítem (Clark, 1973) en una sola regresión, sin la necesidad de agregar los datos.

En ambos experimentos, para abordar

la pregunta de investigación, los modelos incluyeron como predictores cuatro factores; el grupo experimental (i.e., grupo de pacientes con EP, grupo de control etario, grupo de adultos jóvenes), la duración de presentación del prime (SOA de 80 vs 360 ms), la frecuencia léxica del prime (alta vs. baja) y la frecuencia léxica del target (alta vs. baja). Además, se incluyeron en los modelos la interacción entre grupo, SOA y las frecuencias del prime y del target. Debido a que el grupo de interés es la muestra clínica, el promedio global de este grupo sirve como intercepto de la regresión comparando así este grupo directamente con los grupos control. Además, los modelos incluyeron: interceptos aleatorios a nivel de participante e ítem, pendientes aleatorias de SOA tanto para participantes como de grupo y SOA para los ítems, excepto en el modelo de tiempos de reacción con solo SOA como pendiente aleatoria para facilitar convergencia.

\section{Resultados}

\section{Experimento 1}

El porcentaje de respuestas correctas fue considerablemente alto $(m=92 \%)$, sin embargo, dos participantes del grupo control etario fueron excluidos de la muestra ya que evidenciaron un porcentaje de respuestas correctas inferior a $65 \%$. La Figura 2 muestra el patrón de resultados según grupo, SOA, frecuencias léxicas del prime y el target.

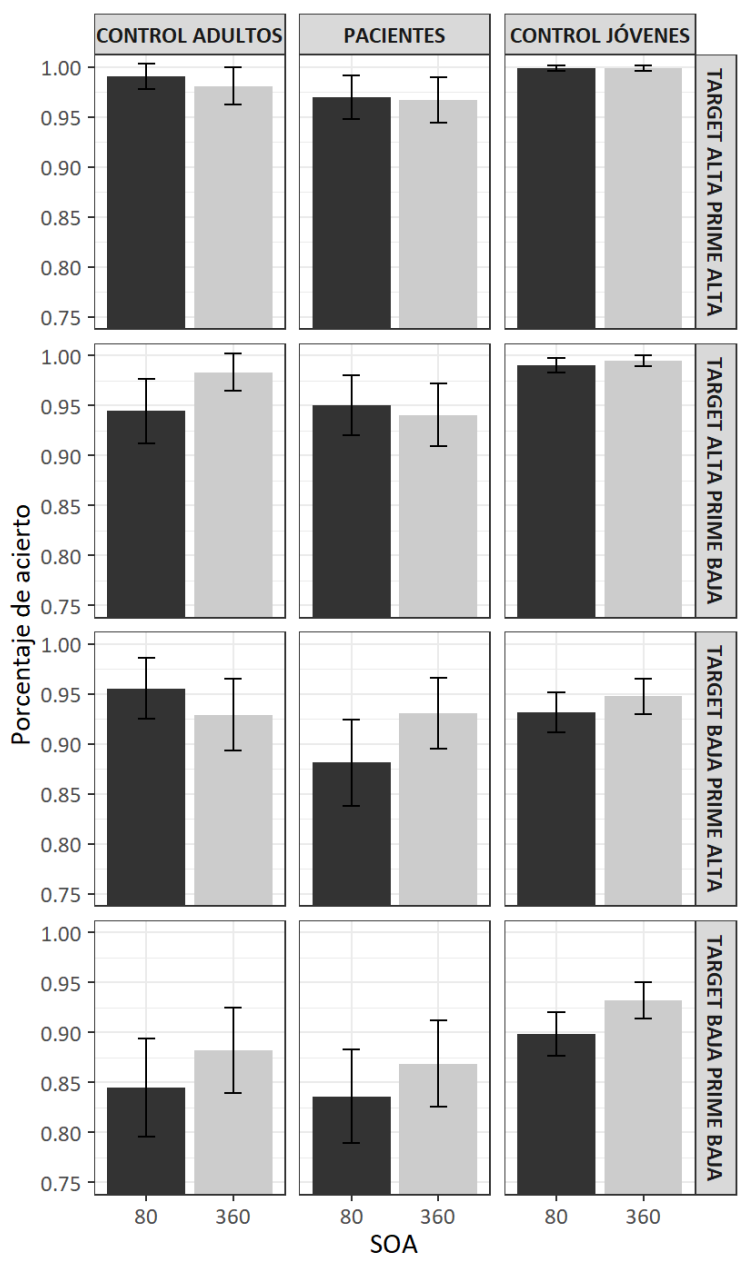

Figura 2. Porcentajes promedio de respuestas correctas divididos en función de grupo (control, pacientes, jóvenes), SOA, y frecuencia léxica del prime y del target en experimento 1. 
Los resultados del análisis de los porcentajes de respuesta a través de la regresión linealgeneralizada aparecen resumidos en la Tabla 1 . Tanto el grupo de adultos jóvenes $(m=0.96$, de=0.19), como el grupo de control etario $(m=0.94$, de $=0.24)$ evidencian un porcentaje de respuestas correctas significativamente superior a la de los pacientes con EP ( $m=0.92$, de=0.28). Por su parte, la frecuencia léxica del prime como del target, inciden en el número de respuestas correctas del grupo de participantes con EP, observándose una disminución acumulativa de las respuestas correctas en función de la frecuencia de las palabras.

Tabla 1

Resultados del análisis de los porcentajes de respuesta mediante la regresión lineal generalizada (experimento 1).

\begin{tabular}{|c|c|c|c|c|c|}
\hline & B & se & z & $\mathrm{p}$ & \\
\hline (Intercepto) & 3.592 & 0.302 & 11.90 & 0.000 & $* * *$ \\
\hline Control Adultos & 1.038 & 0.455 & 2.28 & 0.022 & $*$ \\
\hline Control Jóvenes & 1.975 & 0.426 & 4.64 & 0.000 & $* * *$ \\
\hline SOA & 0.099 & 0.131 & 0.75 & 0.452 & \\
\hline Frecuencia del PRIME & -0.336 & 0.138 & -2.44 & 0.015 & $*$ \\
\hline Frecuencia del TARGET & -0.699 & 0.143 & -4.89 & 0.000 & $* * *$ \\
\hline Control Adultos * SOA & 0.048 & 0.223 & 0.21 & 0.831 & \\
\hline Control Jóvenes * SOA & 0.175 & 0.237 & 0.74 & 0.459 & \\
\hline Control Adultos * Frecuencia del PRIME & -0.160 & 0.221 & -0.72 & 0.471 & \\
\hline Control Jóvenes * Frecuencia del PRIME & -0.010 & 0.246 & -0.04 & 0.967 & \\
\hline Control Adultos * Frecuencia del TARGET & -0.251 & 0.237 & -1.06 & 0.291 & \\
\hline Control Jóvenes * Frecuencia del TARGET & -0.842 & 0.293 & -2.87 & 0.004 & $* *$ \\
\hline SOA * Frecuencia del PRIME & -0.023 & 0.105 & -0.22 & 0.828 & \\
\hline SOA * Frecuencia del TARGET & 0.048 & 0.115 & 0.42 & 0.676 & \\
\hline Control Adultos * SOA * Frecuencia del PRIME & 0.382 & 0.177 & 2.16 & 0.031 & * \\
\hline Control Jóvenes * SOA * Frecuencia del PRIME & 0.084 & 0.136 & 0.62 & 0.536 & \\
\hline Control Adultos * SOA * Frecuencia del TARGET & -0.164 & 0.199 & -0.83 & 0.408 & \\
\hline Control Jóvenes * SOA * Frecuencia del TARGET & -0.035 & 0.228 & -0.15 & 0.877 & \\
\hline
\end{tabular}

$* * *=p<.001 ; * *=p<.01 ; *=p<.05 ; \#=p<.1$

La Figura 3 muestra el patrón de los tiempos de reacción, mientras que la Tabla 2 muestra los resultados del análisis de regresión lineal de efectos mixtos sobre los tiempos de reacción (transformados logarítmicamente) de los tres grupos. 


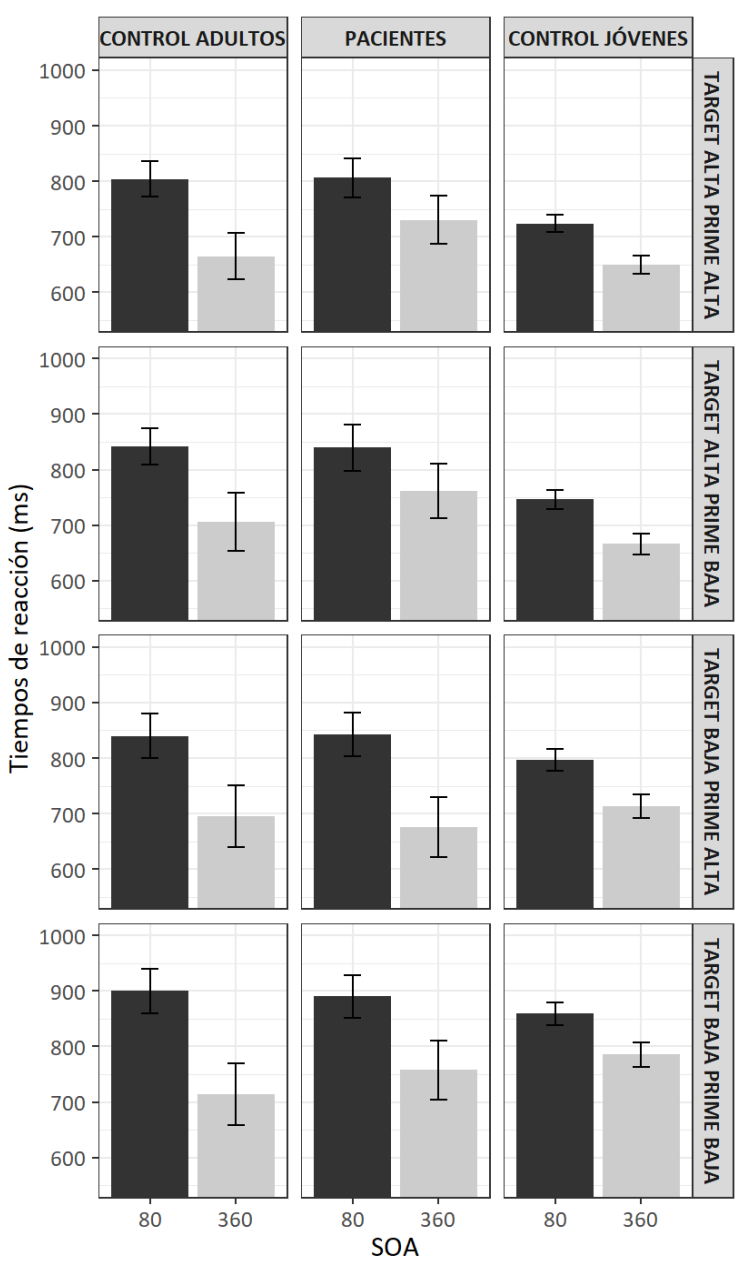

Figura 3. Tiempo de reacción divididos en función de grupo (control, pacientes, jóvenes), SOA, frecuencia léxica del prime y del target en experimento 1.
Los pacientes con EP exhiben un efecto principal de SOA (i.e., respuestas más rápidas para la duración larga del prime), de frecuencia léxica del prime y, marginalmente, de la palabra target (i.e., respuestas más rápidas frente a palabras más frecuentes). Sin embargo, no se observan diferencias generales significativas entre los grupos (efecto principal de grupo en los tiempos de reacción). Por otra parte, se encontró una interacción significativa entre el grupo de adultos jóvenes y los tiempos de presentación del prime. Un efecto similar, pero solo marginalmente significativo, se observa para el grupo de control etario. Esto refleja que el efecto de facilitación del SOA de corta duración es mayor (i.e., tiempos de reacción menores) en el grupo de control etario comparado con los pacientes con EP, sin diferencias entre los tiempos de reacción para los SOA de larga duración. El grupo de adultos jóvenes, por su parte, evidencia tiempos de reacción más cortos para ambos SOAs, pero mayor facilitación relativa para el SOA de corta duración en comparación con los pacientes con EP. 
Tabla 2

Resultados del análisis de regresión lineal de efectos mixtos sobre los tiempos de reacción de los tres grupos (experimento 1).

\begin{tabular}{|c|c|c|c|c|c|}
\hline & b & se & $t$ & $p$ & \\
\hline (Intercepto) & 6.627 & 0.057 & 116.83 & 0.000 & $* * *$ \\
\hline Control Adultos & -0.071 & 0.082 & -0.87 & 0.388 & \\
\hline Control Jóvenes & -0.066 & 0.065 & -1.01 & 0.313 & \\
\hline SOA & -0.120 & 0.020 & -5.91 & 0.000 & $* * *$ \\
\hline Frecuencia del PRIME & 0.051 & 0.011 & 4.74 & 0.000 & $* * *$ \\
\hline Frecuencia del TARGET & 0.019 & 0.011 & 1.76 & 0.079 & $\#$ \\
\hline Control Adultos * SOA & -0.053 & 0.029 & -1.82 & 0.072 & $\#$ \\
\hline Control Jóvenes * SOA & 0.049 & 0.023 & 2.13 & 0.035 & $*$ \\
\hline Control Adultos * Frecuencia del PRIME & -0.021 & 0.011 & -1.84 & 0.065 & $\#$ \\
\hline Control Jóvenes * Frecuencia del PRIME & -0.019 & 0.009 & -2.15 & 0.032 & $*$ \\
\hline Control Adultos * Frecuencia del TARGET & -0.003 & 0.012 & -0.25 & 0.806 & \\
\hline Control Jóvenes * Frecuencia del TARGET & 0.041 & 0.009 & 4.43 & 0.000 & $* * *$ \\
\hline SOA * Frecuencia del PRIME & 0.003 & 0.008 & 0.37 & 0.712 & \\
\hline SOA * Frecuencia del TARGET & -0.008 & 0.008 & -0.92 & 0.360 & \\
\hline Control Adultos * SOA * Frecuencia del PRIME & -0.010 & 0.011 & -0.85 & 0.395 & \\
\hline Control Jóvenes * SOA * Frecuencia del PRIME & -0.001 & 0.009 & -0.09 & 0.932 & \\
\hline Control Adultos * SOA * Frecuencia del TARGET & -0.022 & 0.012 & -1.92 & 0.055 & \# \\
\hline Control Jóvenes * SOA * Frecuencia del TARGET & 0.003 & 0.009 & 0.32 & 0.752 & \\
\hline
\end{tabular}

$* * *=p<.001 ; * *=p<.01 ; *=p<.05 ; \#=p<.1$

De manera similar, el efecto facilitador de la frecuencia es mayor en el grupo de adultos jóvenes. Sin embargo, este dato debe tomarse con cautela ya que tanto la interacción entre el grupo y la frecuencia del prime, como la interacción entre el SOA y el grupo control etario y la frecuencia del prime son marginales.

\section{Experimento 2}

En el experimento 2, el porcentaje de aciertos fue alto $(m=94 \%)$, no obstante, dos participantes del grupo control etario fueron excluidos de la muestra tras evidenciar un porcentaje de respuestas correctas inferior a $65 \%$. 


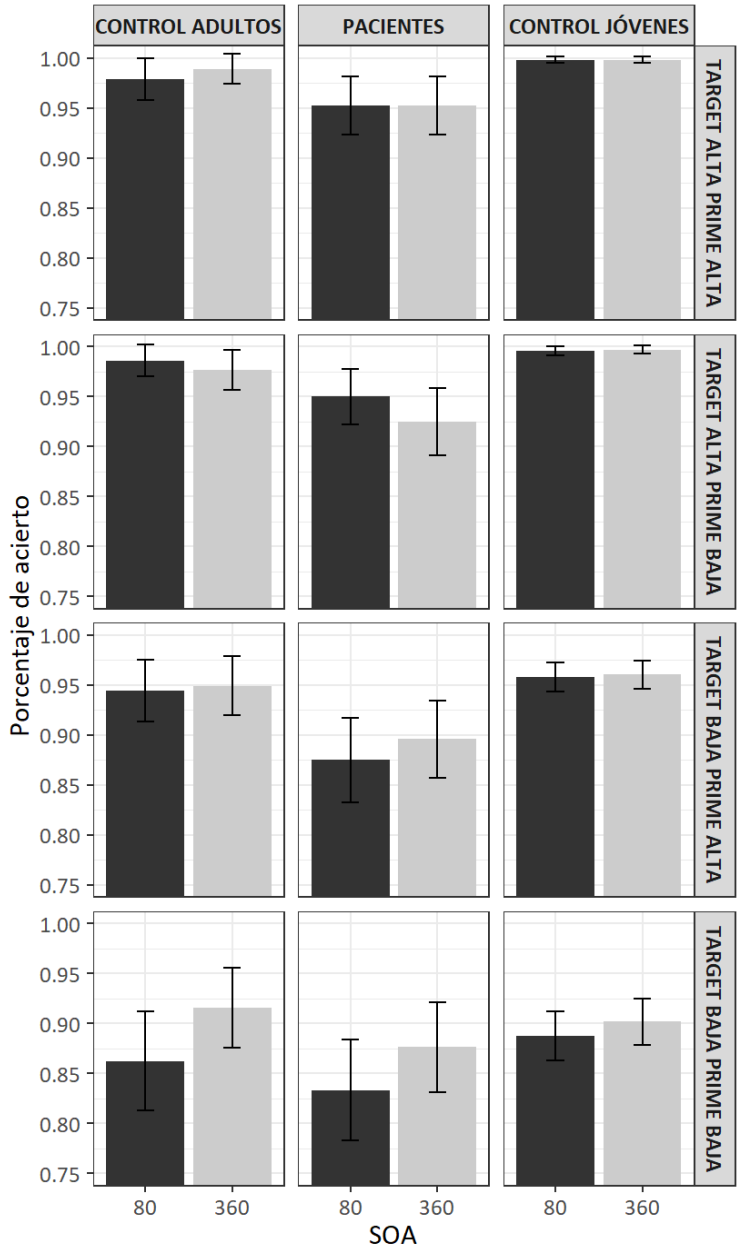

Figura 4. Porcentajes promedio de respuestas correctas divididos en función de grupo (control, pacientes, jóvenes), SOA, frecuencia léxica del prime y del target en experimento 2 .
Los resultados del análisis de los porcentajes de respuesta correctas a través de la regresión lineal generalizada para el experimento 2 aparecen resumidos en la Tabla 3. Tanto el grupo de adultos jóvenes ( $m=0.96$, de $=0.18$ ) como el grupo de control etario ( $m=0.95$, de $=0.21$ ) muestran un porcentaje de respuesta correctas significativamente superior a la de los pacientes con EP ( $m=0.90, d e=0.29)$. Además, se observa un efecto significativo de la frecuencia del target, el cual refleja un mayor acierto para las frecuencias léxicas altas que las bajas. Finalmente, se encuentra una interacción entre el grupo de adultos jóvenes y el efecto de frecuencia léxica de la palabra target, probablemente provocada por el efecto techo de las respuestas de los jóvenes sobre las palabras de alta frecuencia. 
Tabla 3

Resultado del análisis de los porcentajes de respuesta correctas mediante la regresión lineal generalizada (experimento 2).

\begin{tabular}{|c|c|c|c|c|c|}
\hline & b & se & $z$ & $p$ & \\
\hline (Intercepto) & 3.393 & 0.291 & 11.68 & 0.000 & $* * *$ \\
\hline Control Adultos & 1.010 & 0.412 & 2.45 & 0.014 & $*$ \\
\hline Control Jóvenes & 2.749 & 0.467 & 5.89 & 0.000 & $* * *$ \\
\hline SOA & 0.037 & 0.118 & 0.32 & 0.752 & \\
\hline Frecuencia del PRIME & -0.031 & 0.145 & -0.21 & 0.834 & \\
\hline Frecuencia del TARGET & -0.562 & 0.153 & -3.68 & 0.000 & $* * *$ \\
\hline Control Adultos * SOA & 0.086 & 0.211 & 0.41 & 0.683 & \\
\hline Control Jóvenes * SOA & 0.069 & 0.270 & 0.25 & 0.800 & \\
\hline Control Adultos * Frecuencia del PRIME & -0.142 & 0.177 & -0.80 & 0.422 & \\
\hline Control Jóvenes * Frecuencia del PRIME & 0.033 & 0.249 & 0.13 & 0.894 & \\
\hline Control Adultos * Frecuencia del TARGET & -0.236 & 0.203 & -1.16 & 0.246 & \\
\hline Control Jóvenes * Frecuencia del TARGET & -1.224 & 0.371 & -3.30 & 0.001 & $* * *$ \\
\hline SOA * Frecuencia del PRIME & 0.049 & 0.101 & 0.49 & 0.627 & \\
\hline SOA * Frecuencia del TARGET & -0.077 & 0.112 & -0.69 & 0.492 & \\
\hline Control Adultos * SOA * Frecuencia del PRIME & -0.012 & 0.169 & -0.07 & 0.945 & \\
\hline Control Jóvenes * SOA * Frecuencia del PRIME & -0.016 & 0.137 & -0.12 & 0.905 & \\
\hline Control Adultos * SOA * Frecuencia del TARGET & 0.194 & 0.207 & 0.94 & 0.348 & \\
\hline Control Jóvenes * SOA * Frecuencia del TARGET & 0.043 & 0.269 & 0.16 & 0.874 & \\
\hline
\end{tabular}

$* * *=p<.001 ; * *=p<.01 ; *=p<.05 ; \#=p<.1$ 
La Figura 3 muestra el patrón de los tiempos de reacción en el experimento 2 . Por su parte, la Tabla 4 presenta los resultados del análisis de los tiempos de reacción transformados logarítmicamente a través de un modelo de regresión lineal de efectos mixtos para el presente experimento.

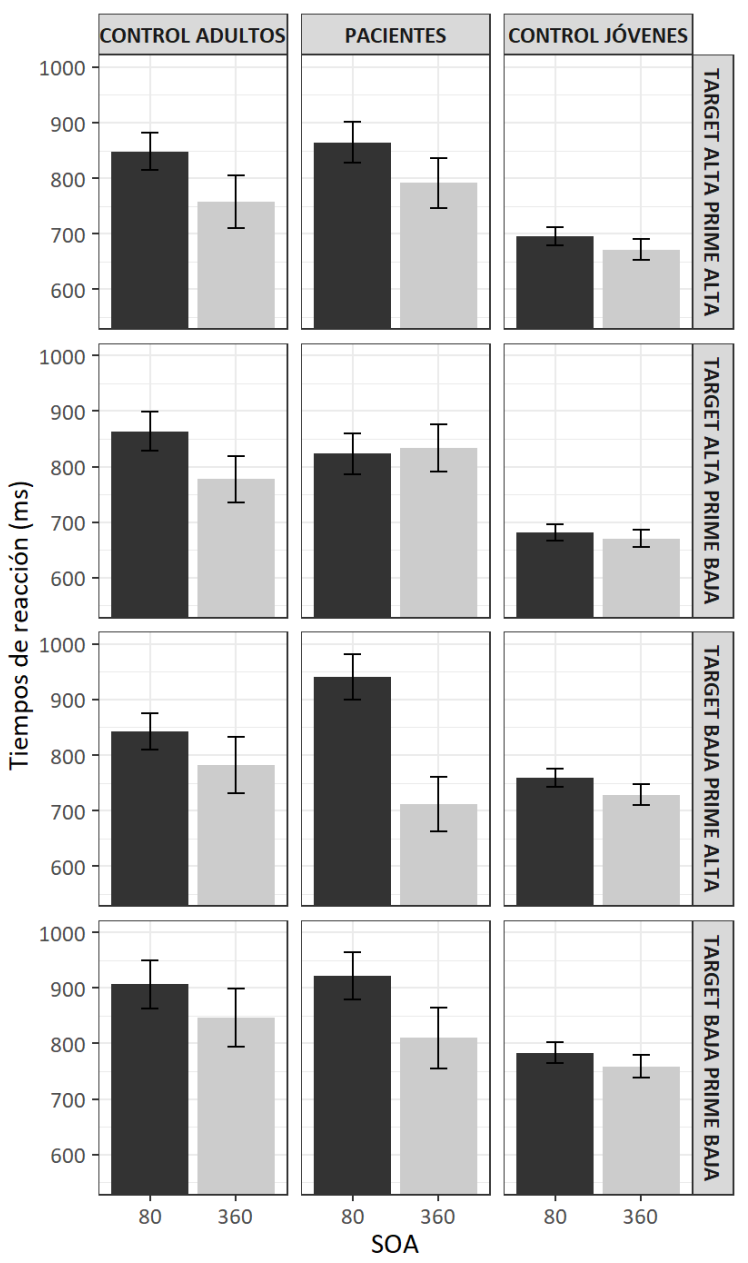

Figura 5. Tiempo de reacción divididos en función de grupo (control, pacientes, jóvenes), SOA, frecuencia léxica del prime y del target en experimento 2.
Los pacientes con EP exhiben efectos significativos del SOA (i.e., respuestas más rápidas para la duración larga del prime), de las frecuencias léxicas del prime y de la palabra target (i.e., respuestas más rápidas frente a palabras más frecuentes), aunque este último es marginal. Además, el grupo experimental se mostró significativamente más lento que el grupo de adultos jóvenes en sus tiempos de respuesta. A esto se suma un efecto distinto de los tiempos de presentación del prime, así como de la frecuencia léxica de la palabra target sobre el grupo de adultos jóvenes y el grupo clínico. El efecto de facilitación del SOA de larga duración es mayor en el grupo experimental que en los adultos jóvenes, mientras que el de frecuencia del target es mayor para adultos jóvenes comparado con los pacientes. La interacción entre la frecuencia del prime y su duración también tiene un efecto distinto en el grupo de interés que en el grupo de adultos jóvenes. Así, el efecto facilitador de la duración larga es mayor en primes de alta frecuencia en participantes con EP, mientras que en los adultos jóvenes no se encontraron estos efectos. 
Tabla 4

Resultado del análisis de los tiempos de reacción a través de un modelo de regresión lineal de efectos mixtos (experimento 2).

\begin{tabular}{|c|c|c|c|c|c|}
\hline & b & se & $t$ & $p$ & \\
\hline (Intercepto) & 6.701 & 0.055 & 122.90 & 0.000 & $* * *$ \\
\hline Control Adultos & -0.065 & 0.078 & -0.83 & 0.409 & \\
\hline Control Jóvenes & -0.166 & 0.062 & -2.66 & 0.009 & $* *$ \\
\hline SOA & -0.092 & 0.017 & -5.40 & 0.000 & $* * *$ \\
\hline Frecuencia del PRIME & 0.019 & 0.010 & 1.89 & 0.061 & $\#$ \\
\hline Frecuencia del TARGET & 0.026 & 0.010 & 2.47 & 0.014 & $*$ \\
\hline Control Adultos * SOA & -0.003 & 0.024 & -0.14 & 0.887 & \\
\hline Control Jóvenes * SOA & 0.064 & 0.019 & 3.30 & 0.001 & $* *$ \\
\hline Control Adultos * Frecuencia del PRIME & 0.008 & 0.010 & 0.87 & 0.384 & \\
\hline Control Jóvenes * Frecuencia del PRIME & -0.006 & 0.008 & -0.82 & 0.410 & \\
\hline Control Adultos * Frecuencia del TARGET & 0.009 & 0.010 & 0.90 & 0.366 & \\
\hline Control Jóvenes * Frecuencia del TARGET & 0.030 & 0.008 & 3.78 & 0.000 & $* * *$ \\
\hline SOA * Frecuencia del PRIME & 0.025 & 0.007 & 3.32 & 0.001 & $* * *$ \\
\hline SOA * Frecuencia del TARGET & -0.015 & 0.008 & -1.95 & 0.051 & \# \\
\hline Control Adultos * SOA * Frecuencia del PRIME & -0.011 & 0.010 & -1.15 & 0.249 & \\
\hline Control Jóvenes * SOA * Frecuencia del PRIME & -0.020 & 0.008 & -2.58 & 0.010 & $* *$ \\
\hline Control Adultos * SOA * Frecuencia del TARGET & 0.008 & 0.010 & 0.79 & 0.431 & \\
\hline Control Jóvenes * SOA * Frecuencia del TARGET & 0.013 & 0.008 & 1.63 & 0.104 & \\
\hline
\end{tabular}

$* * *=p<.001 ; * *=p<.01 ; *=p<.05 ; \#=p<.1$

Finalmente, la interacción entre el tiempo de presentación del prime y la frecuencia tanto del prime como del target, modularon las latencias del grupo experimental, pero de manera opuesta. Así, el efecto facilitador del SOA de larga duración es mayor para los primes de alta frecuencia, el mismo efecto es mayor para target de baja frecuencia.

\section{Discusión general}

El impacto de la EP en el procesamiento del lenguaje ha cobrado gran interés durante las últimas décadas, ya que su estudio ha permitido extraer conclusiones importantes sobre los mecanismos neurales responsables de las alteraciones a distintos niveles lingüísticos (Bastiaanse \& Leenders, 2009; Colman \& Bastiaanse, 2011).

En este ámbito, las alteraciones en el procesamiento semántico han sido respaldadas por un número de estudios que han abordado el impacto de la EP en la recuperación del significado y en la propagación de información en tareas de 
reconocimiento visual de palabras (Angwin et al., 2005; Angwin et al., 2006; Angwin et al., 2007). El presente estudio contribuye a comprender mejor este fenómeno, a partir de la investigación de los efectos de la frecuencia léxica (tanto del prime como del target) y la mediación indirecta (orto-fonológica) sobre el procesamiento semántico. En particular sobre los tiempos de respuesta en tareas de reconocimiento visual de palabras (Grossman, 1999; Copland et al., 2009; Zanini, Tavano \& Fabbro, 2010; Herrera, Rodríguez \& Cuetos, 2012).

Bajo estas consideraciones, los hallazgos de la presente investigación han confirmado que existe un procesamiento semántico alterado como consecuencia directa de la enfermedad e independiente de las características propias del envejecimiento cognitivo (Véliz, Riffo \& Arancibia, 2010; Véliz, 2014). No obstante, las variables léxicas (frecuencia de palabras) e intervalos temporales entre el prime y el target (SOA) afectan el acceso léxico a palabras escritas en pacientes con EP.

Los resultados del experimento 1 muestran diferencias importantes entre el grupo de pacientes y el grupo control de adultos jóvenes, tanto en el acierto como en el tiempo de sus respuestas. Los hallazgos de mayor interés son aquellas diferencias dadas entre el grupo de pacientes y el grupo de control etario. En este sentido, los participantes con EP cometen en general más errores que sus pares sin EP. Además, sus aciertos son modulados de manera distinta por la frecuencia del prime y el tiempo de presentación. Sin embargo, los tiempos de presentación y la frecuencia del prime, así como la interacción entre los tiempos de presentación del prime y la frecuencia del target modulan los tiempos de respuesta de manera solo marginalmente (en términos estadísticos) distinta en los pacientes con EP en comparación a sus pares sanos.

Los pacientes muestran efectos significativos tanto de la frecuencia léxica del prime como del target, ejerciendo una influencia directa en el número de respuestas correctas, como es esperable en participantes jóvenes y adultos mayores saludables. Lo anterior sugiere que, a pesar del déficit semántico evidenciado por los pacientes, siguen siendo sensibles a las variaciones de frecuencia léxica, las cuales suponen mayor dificultad cuando los estímulos son de baja frecuencia (De Vega \& Cuetos, 1999; Cuetos, González \& Cuetos, 2012). Además, el efecto combinado de la frecuencia del prime y el tiempo de presentación es distinto en los pacientes con EP que en el grupo de control etario; solo estos últimos son sensibles a la frecuencia del prime cuando la presentación de éste es breve.

No obstante, la frecuencia del target tiene un efecto distinto sobre el porcentaje de respuestas correctas del grupo clínico y el de adultos jóvenes. Ello, debido, probablemente, a que la baja frecuencia del target no tiene un efecto tan importante en el grupo de jóvenes, como lo tiene en el grupo clínico. Finalmente, el efecto de la frecuencia del prime es más fuerte en el SOA de corta duración en el grupo de control etario, mientras que este mismo efecto aparece más marcadamente en el SOA de larga duración en el grupo de pacientes con EP.

En el experimento 2, ambos grupos control superan nuevamente a los pacientes con EP en el número de respuestas correctas, pero en contraste con el primer experimento, solo la frecuencia del target afecta esta medida. De manera similar al grupo 
control, la frecuencia del prime no afecta la precisión de las respuestas del grupo de pacientes, posiblemente ya que el prime media indirectamente al target. Por tanto, es el vecino orto-fonológico del prime el que modula el acceso a la palabra target.

Estos resultados son consistentes con estudios previos que muestran dificultades semánticas en pacientes con EP (Angwin, Chenery, Copland, Murdoch \& Silburn, 2005; Mari-Beffa, Hayes, Machado \& Hidle, 2005; Arnott et al., 2011), y que dichas dificultades no estarían asociadas de manera directa a mecanismos automáticos o más conscientes de manera particular. Ello porque la precisión de respuestas no es modulada por el SOA (Waters \& Patel, 1999; Portin, Laatu, Revonsuo \& Rinne, 2000). Sin embargo, los resultados muestran que los efectos de la frecuencia del prime son sensibles a procesos conscientes, pero no automáticos, ya que solo aparecen claramente con una duración prolongada del prime.

Al igual que el número de respuestas correctas, los tiempos de reacción en el experimento 1 son afectados por la frecuencia del prime y del target (marginalmente). En contraste, la velocidad de respuesta sí parece estar directamente ligada a procesos automáticos vs. conscientes (efecto de SOA). Esto puede ser explicado en función de la propagación de la activación a nivel semántico y de la integridad del procesamiento controlado y el procesamiento consciente del significado (Angwin, Chenery, Copland, Murdoch \& Silburn, 2007). En el experimento 2, también se observaron efectos de SOA y frecuencia léxica sobre los tiempos de reacción del grupo clínico. El efecto de la frecuencia del prime es particularmente fuerte para las palabras de alta frecuencia cuando existe una duración larga del prime. Estos resultados sugieren nuevamente que el proceso de acceso consciente y controlado está más intacto en la EP, mientras que los procesos de acceso léxico automático parecen estar más afectados.

En síntesis, los resultados de este estudio muestran que los efectos de priming semántico están modulados por la frecuencia léxica y el tipo de SOA, aunque sus efectos son distintos en los grupos comparados. En este sentido, la frecuencia léxica del prime favorece el desempeño del grupo clínico en el SOA largo, lo cual puede ser explicado en función de la integridad del procesamiento consciente, pero no así del procesamiento automático. Los efectos del priming mediado orto-fonológicamente, en cambio, reflejan una disminución de la activación de la información ortográfica en los intervalos entre prime y target, aunque la interacción entre la frecuencia del prime y del target junto con el tipo de SOA presentan patrones similares a los observados en la condición de priming semántico. Los resultados obtenidos respaldan el impacto de la EP en el procesamiento semántico durante el reconocimiento visual de palabras en tareas de priming asociado semánticamente y en tareas de priming semántico mediado orto-fonológicamente.

Financiamiento: Beca de Doctorado Nacional Conicyt, 2015. Folio 21150571.

\section{Referencias}

Altmann, L. y Troche, M. (2011). High-Level Language Production in Parkinson's Disease: A Review. Parkinson's Disease, 2011, 1-12. doi: 10.4061/2011/238956

Álvarez, C., De Vega, M. \& Carreiras, M. (1998). La sílaba 
como unidad de activación léxica en la lectura de palabras trísilábicas. Psicothema, 10, 371-386.

Angwin, A., Chenery, H., Copland, D., Murdoch, B. \& Silburn, P. (2005). Summation of semantic priming and complex sentence comprenhension in Parkinson's disease. Cognitive Brain Research, 25, 78-89. doi: 10.1016/j.cogbrainres.2005.04.008

Angwin, A., Chenery, H., Copland, D., Murdoch, B. \& Silburn, P. (2006). The influence of dopamine on semantic activation in Parkinson's disease: evidence from a multipriming task. Neuropsychology, 20, 299-306. doi: 10.1037/08944105.20.3.299

Angwin, A., Chenery, H., Copland, D., Murdoch, B. \& Silburn, P. (2007). The speed of lexical activation is altered in Parkinson's disease. Journal of Clinical and Experimental Neuropsychology, 29, 73-85. doi: $10.1080 / 13803390500507188$

Arnott, W., Copland, D., Chenery, H., Murdoch, P., Silburn, P. \& Angwin, A. (2011). The influence of dopamine on automatic and controlled semantic activation in Parkinson's Disease. Parkinson's Disease, 2011, 1-11. doi: 10.4061/2011/157072

Balota, D. A., \& Chumbley, J. I. (1984). Are lexical decisions a good measure of lexical access? The role of wordfrequency in the neglected decision stage. Journal of Experimental Psychology: Human Perception and Performance,10, 340-357. doi: 10.1037//00961523.10.3.340

Bastiaanse, R. \& Leenders, K. (2009). Language and Parkinson's Disease. Cortex, 45, 912-914. doi: 10.1016/j.cortex.2009.03.011

Bates, D., Maechler, M., Bolker, B., Walker, S. (2015). Fitting Lineal Mixed-Effects Models Using Ime4. Journal of Statistical Software, 67, 1-48. doi:10.18637/jss.v067.i01

Brysbaert, M., Mandera, P., \& Keuleers, E. (2018). The word frequency effect in word processing: An updated review. Current Directions in Psychological Science, 27, 45-50. doi: $10.1177 / 0963721417727521$

Cardona, J., Gershanik, O., Gelormini-Lezama, C., Cardona, S., Kargieman, L., Trujillo, N., Arévalo, A., Amoruso, L., Manes, F. e Ibáñez, A. (2013). Action-verb processing in Parkinsons's Disease: new pathways for motor-language coupling. Brain Structure and Function, 218, 1355-1373. doi: 10.1007/s00429-013-0510-1
Carreiras, M. \& Perea, M. (2002). Masked Priming Effects With Syllabic Neighbors in a Lexical decistion Task. Journal of Experimental Psychology, 28, 1228-1242. doi: 10.1037//0096-1523.28.5.1228

Castner, J., Copland, D., Silburn, P., Coyne, T., Sinclair, F. \& Chenery, H. (2007). Lexical-semantic inhibitory mechanisms in Parkinson's disease as a function of subthalamic stimulation. Neuropsychologia, 45, 3167-3177. doi: 10.1016/j.neuropsychologia.2007.06.019

Clark, H. (1973). The language-as-fixed-effect fallacy: A critique of language statistics in psychological research. Journal of Verbal Learning and Verbal Behavior, 12, 335359. doi: 10.1016/s0022-5371(73)80014-3

Colman, K. \& Bastiaanse, R. (2011). Language Processing in Parkinson's Disease Patients Without Dementia. En J. Dushanova (Ed.): Diagnostics and Rehabilitations of Parkinson's Disease (pp. 165-188). Croatia: InTech. doi: $10.5772 / 18035$

Copland, D., Sefe, G., Ashley, J., Hudson, C. \& Chenery, H. (2009). Impaired semantic inhibition during lexical ambiguity repetition in Parkinson's disease. Cortex, 45, 943949. doi: 10.1016/j.cortex.2009.02.023

Copland, D., McMahon, P., Silburn, P. \& Zubicaray, G. (2009). Dopaminergic Neuromodulation of Semantic Processing: A 4-T fMRI Study with Levodopa. Cerebral Cortex,19, 2651-2658. doi: 10.1093/cercor/bhp017

Cuetos, F., González, J. \& de Vega, M. (2018). Psicología del lenguaje. Madrid: Editorial Médica Panamericana.

Davis, C. \& Perea, M. (2005). BuscaPalabras: A program for deriving orthographic and phonological neighborhood statistics and other psycholinguistic indices in Spanish. Behavior Reasearch Methods, 37, 665-671. doi: 10.3758/bf03192738

De Vega, M. \& Cuetos, F. (1999). Psicolingüística del Español. Madrid: Editorial Trolta.

Duñabeitia, J., Carreiras, M. \& Perea, M. (2008). Are coffee and toffee served in a cup? Ortho-phonologically mediated associative priming. The Quarterly Journal of Experimental Psychology, 61, 1861-1872. doi: 10.1080/17470210701774283

Elorriaga, S., Tapia, A., Rodríguez, M., Carrasco, H., Fernández, T. \& Silva, J. (2012). Phonological processing during reading: A priming study in patients with Parkinson's 
disease. Journal of Behavior, Health \& Social Issues, 4, 4558. doi: 10.5460/jbhsi.v4.2.34107

Fischler, I. (1977). Semantic facilitation without association in a lexical decision task. Memory \& Cognition, 5, 335-339. doi:10.3758/BF03197580

Garman, M. (1995). Psicolingüística. Madrid: Visor Libros.

Grossman, M. (1999). Sentence processing in Parkinson's disease. Brain and Cognition, 40, 387-413. doi: 10.1006/brcg.1999.1087

Heaton, R. K., Chelune, G. J., Talley, J. L., Kay, G. G. \& Curtis, G. (1993). WCST: Test de clasificación de tarjetas de Wisconsin. Madrid: TEA.

Helmich, R., Hallet, M., Deuschl, G., Toni, I. \& Bloem, B. (2012). Cerebral causes and consequences of Parkinsonian resting tremor: a tale of two circuits? Brain: A Journal of Neurology, 135, 3206-3226. doi: 10.1093/brain/aws023

Herrera, E., Rodríguez, J. \& Cuetos, F. (2012). The effect of motion content in action naming by Parkinson's disease patiens. Cortex, 48, 900 - 904. doi: 10.1016/j.cortex.2010.12.007

Illes, J., Metter, E.J., Hanson, R. \& Iritani, S. (1988). Language Production in Parkinson's Disease: Acoustic and Linguistic Consideration. Brain and Language, 33, 146-160. doi: 10.1016/0093-934x(88)90059-4

Kuznetsova, A., Brockhoff, P.B., Christensen, R.H.B. (2017). ImerTest Package: Tests in Lineal Mixed Effects Models. Journal of Statistical Software, 82, 1-26. doi: 10.18637/jss.v082.i13

Mari-Beffa, P., Hayes, A., Machado, L., \& Hindle, J. (2005). Lack of inhibition in Parkinson's disease: Evidence from a lexical decision task. Neusopsychologia, 43, 638-646. doi: 10.1016/j.neuropsychologia.2004.

Monsell, S. (1991). The nature and locus of word frequency effects in reading. In D. Besner \& G. W. Humphreys (Eds.), Basic processes in reading: Visual word recognition (pp.148197). Hillsdale, NJ: Erlbaum.

Nadeau, S. (2008). Subcortical Language Mechanisms. En: B. Stemmer \& H. Whitaker (Eds.). Handbook the Neuroscience of Language (pp. 329-338). London: Elsevier.

Perea, M. \& Rosa, E. (2000). The effects of orthographic neighborhood in reading and laboratory word identification tasks: A review. Psicológica, 21, 327-340.
Portin, R., Laatu, S., Revonsuo, A. \& Rinne, U. (2000). Impairment of semantic knowledge in Parkinson Disease. Archives of Neurology, 57, 1338- 1343 . doi: 10.1001/archneur.57.9.1338

Rayner, K. \& Duffy, S. (1986). Lexical complexity and fixation time in reading: Effects of word frequency, verb complexity, and lexical ambiguity. Memory and Cognition, 14, 191-201. doi: 10.3758/bf03197692

Rey, A. (1997). Rey: Test de copia y de reproducción de memoria de figuras geométricas complejas. Madrid: TEA Ediciones.

R Core Team. (2017). R: A language and environment for statistical computing. $\mathrm{R}$ Foundation for Statistical Computing, Vienna, Austria. https://www.r-project.org

Ting Lu, Y., Neng Chang, W., Chih Chang, C., Hsien Lu, C., Ching Chen, N., Wei Huang, C., Che Lin, W. \& Ting Chang, Y. (2016) Insula volume and salience network are associated with memory decline in Parkinson's Disease: complementary analyses of Voxel-Based Morphometry versus Volume of Interest. Parkinson's Disease, 2016, 1-8. doi: $10.1155 / 2016 / 2939528$

Tirapu, J., Muñoz, J. \& Pelegrín, C. (2002). Funciones ejecutivas: necesidad de una integración conceptual. Revista de Neurología, 34, 673-685. doi: 10.33588/rn.3407.2001311

Tirapu, J. \& Muñoz, J. (2005). Memoria y funciones ejecutivas. Revista de Neurología, 41, 475-484. doi: 10.33588/rn.4108.2005240

Véliz, M., Riffo, B. \& Arancibia, B. (2010). Envejecimiento cognitivo y procesamiento del lenguaje: Cuestiones relevantes. Revista de Lingüística Teórica y aplicada, 48, 75103. doi: $10.4067 /$ s0718-48832010000100005

Véliz, M. (2014). Efectos de envejecimiento en los procesos de comprensión y producción del lenguaje. Paideia, 54, 1132.

Walsh, B. y Smith, A. (2011). Linguistic Complexity, Speech Production, and Comprehension in Parkinson's Disease: Behavioral and Physiological Indices. Journal of Speech, Language, and Hearing, 54, 1-29. doi: 10.1044/10924388(2010/09-0085)

Watters, P. \& Patel, M. (1999). Semantic processing deficits in patiens with Parkinson's disease: degraded representation or defective retrieval? Revue de Psychiatrie 
et de Neuroscience, 24, 322-332.

Wechsler, D. (2012). WAIS-IV. Escala de inteligencia de Wechsler para adultos IV. Manual de aplicación y corrección. Madrid: NCS Pearson, Inc.

Zanini, S., Tavano, A. \& Fabbro, F. (2010). Spontaneous language production in bilingual Parkinson's disease patients: Evidence of greater phonological, morphological and syntactic impairments in native language. Brain \& Language, 113, 84-89. doi: 10.1016/j.bandl.2010.01.005 